\title{
Battery Swallow-Induced Tracheoesophageal Fistula in a Pediatric Patient
}

\author{
Lindsey Nguyen ${ }^{1}$, Sathish Ramanathan ${ }^{2}$, Hamid Vahabzadeh-Monshie ${ }^{2}$, Jack Borders ${ }^{2}$, and \\ Alberto de Armendi ${ }^{2}$ \\ ${ }^{1}$ The University of Oklahoma College of Medicine \\ ${ }^{2}$ The University of Oklahoma Health Sciences Center
}

October 31, 2020

\begin{abstract}
Tracheoesophageal fistula (TEF) is an abnormal connection between trachea and esophagus. Acquired TEF arise from trauma, caustic fluids ingestion, and iatrogenic injuries (ventilation, esophageal atresia repair, radiation, tracheal/esophageal stents). We discuss diagnosis, anesthetic management, and surgical correction of battery swallow-induced TEF, not previously reported in pediatric anesthesia and otorhinolaryngology literatures.
\end{abstract}

Title: Battery Swallow-Induced Tracheoesophageal Fistula in a Pediatric Patient

Running Title: Battery Swallow-Induced TEF

\section{Authors:}

Lindsey N. Nguyen, BA ${ }^{1}$

Sathish Ramanathan, $\mathrm{MD}^{2}$

Hamid R. Vahabzadeh-Monshie, $\mathrm{MD}^{2}$

Jack C. Borders, $\mathrm{MD}^{2}$

Alberto J. de Armendi, MD, PhD, $\mathrm{MBA}^{2}$

\section{Corresponding Author:}

Alberto J. de Armendi, MD, PhD, MBA

University of Oklahoma Health Sciences Center, Department of Anesthesiology

920 Stanton L Young Blvd, Suite 1140, Oklahoma City, Oklahoma 73104

Fax: (405) 271-4015

Telephone: (405) 271-4351

E-mail: Alberto-dearmendi@ouhsc.edu

\section{Institutions:}

${ }^{1}$ University of Oklahoma College of Medicine, Oklahoma City, Oklahoma, USA

${ }^{2}$ University of Oklahoma Health Sciences Center, Departments of Anesthesiology and Otolaryngology, Oklahoma City, Oklahoma, USA 
Keywords: tracheoesophageal fistula, battery, airway anomaly, pediatric, anesthesia

\section{Key Clinical Message:}

Battery swallow-induced TEF is a rare, life-threatening condition with increasing incidence requiring adequate preparation, understanding of TEF anatomy, and excellent communication among all involved in the care of these pediatric critical airways.

\section{Summary:}

Tracheoesophageal fistula (TEF) is an abnormal connection between the trachea and esophagus. Rare acquired TEF arises from trauma, caustic fluids ingestion, and iatrogenic injuries (ventilation, esophageal atresia repair, radiation, or tracheal/esophageal stents). ${ }^{1}$ We discuss the diagnosis, anesthetic management, and surgical correction of battery swallow-induced TEF, not previously reported in the pediatric anesthesia and otorhinolaryngology literatures.

\section{Introduction:}

Etiology of tracheoesophageal fistula is commonly congenital due to failure of proper embryonic lung bud branching, resulting in lateral septation of the foregut into the esophagus and trachea. Clinically, TEF severity relates to oxygenation and aspiration pneumonia from gastric reflux.

Acquired TEF is rare. Battery ingestion has recently increased in incidence, presenting asymptomatically or with nonspecific symptoms of fever, poor feeding, and dyspnea. The battery establishes an electrical circuit within the esophagus producing hydroxide ions at the negative pole forming alkali burns that perforate the esophagus and create a TEF. ${ }^{2}$ Treatment of battery swallow-induced TEF requires immediate surgery, preferably within two hours to minimize friability, tissue necrosis, fistula enlargement, tracheobronchial contamination, sepsis, and nutritional problems. ${ }^{3}$ We discuss the diagnosis, anesthetic approach, and surgical corrections of battery swallow-induced TEF (Figure 1) in a pediatric patient.

\section{Case Report:}

A 13-month-old patient $(8.9 \mathrm{~kg})$ post-esophagoscopy and button battery removal presented for surgical repair of $30 \%$ complete tracheal erosion from cervical 6-thoracic 1. Past medical history included recent upper respiratory tract infection, resolving viral myocarditis, sick euthyroid syndrome, and salt wasting syndrome. Pre-operatively, the critical airway was at risk for life-threatening tracheal separation, bleeding (if aorto-esophageal fistula formed), and mediastinitis. Physical examination showed stable vital signs with diminished air entry to left lung. Chest x-ray demonstrated right upper lobe atelectasis and left lung base airspace opacity.

The patient arrived to the operating room already intubated in stable condition and was premedicated with midazolam and fentanyl. General anesthesia was induced with cis-atracurium, fentanyl, and propofol. Under direct vision, the otolaryngologist extubated the trachea. Albuterol and epinephrine were administered for bronchodilation. After encountering ventilation difficulty from bloody secretions, a 3.5 cuff tracheostomy tube was successfully placed.

One week later, the patient underwent direct laryngoscopy/bronchoscopy and TEF repair. General anesthesia was induced with rocuronium, fentanyl, and propofol. Total intravenous anesthesia was maintained with propofol infusion and fentanyl boluses. Upon conclusion of surgery, the patient was awakened with trachea extubated uneventfully. The patient recovered without respiratory or cardiovascular complications.

\section{Discussion:}

Tracheoesophageal fistula is an abnormal communication between trachea and esophagus. A recent common cause of acquired TEF is battery ingestion. Risks involved with TEF surgical ligation are associated with fragile necrotic tissue, including fistula formation, tracheal separation, pneumothorax, pneumomediastinum, esophageal stricture, vocal cord paralysis, thyroid hemorrhage, spine spondylodiscitis, bleeding (if aorto-esophageal fistula formed), chest infections/sepsis, and death. ${ }^{4}$ Anesthetic management challenges are 
endotracheal tube (ETT) placement failure if TEF size and position are unknown, post-operative airway obstruction, aspiration, and loss of airway from bleeding or difficulty with ETT replacement. Anesthesia safety precautions for battery swallow-induced TEF rely on understanding size and position of the TEF pre-operatively.

Battery removal is essential as severity of damage and complications correlate to duration of battery lodgment in the esophagus. ${ }^{5}$ If surgery is not readily available, mitigating efforts to increase $\mathrm{pH}$ at the battery site include drinking weakly acidic liquid (lemon/orange juice), honey, sucralfate, and dilute acetic acid. ${ }^{5}$ Direct laryngoscopy/bronchoscopy should be performed to confirm TEF, followed by surgical repair. ${ }^{3}$ Conservative management with temporary endoluminal tracheal or esophageal stents has been implemented to prevent pulmonary contamination. ${ }^{1}$ Surgical management of acquired TEF involves single-stage patch repair of the tracheal and esophageal defects with possible partial esophagectomy, cervical esophagostomy, and gastrostomy. Utilization of vascularized local tissue flaps has been recommended to reinforce repair sites. Postoperatively, intravenous antibiotics are administered if concerned for mediastinitis, tracheal stents inserted to retain airway patency, and gastrostomy feeds implemented for nutritional support. ${ }^{3}$ Early extubation and avoidance of post-surgical positive-pressure ventilation have been recommended. ${ }^{1}$

The Button Battery Task Force aims to decrease the incidence of battery ingestion in children. Despite concerted efforts from government, industry, academia, medicine, and public health, battery ingestion continues to occur. ${ }^{5}$ The National Capital Poison Center (website: http://www.poison.org/battery, accessed April 1, 2020) and National Battery Ingestion Hotline (1-800-498-8666, available 24 hours/7 days a week) guide the public in battery ingestion issues.

In conclusion, we present a life-threatening battery swallow-induced TEF, which required emergent anesthesia for infection drainage and placement of ETT distal to the damage for TEF repair. We emphasize preparation, understanding TEF anatomy, and excellent communication amongst surgeons, radiology, blood bank, and anesthesiologists for the proper care of these pediatric critical airways.

Acknowledgements: None.

Conflict of Interest: None.

\section{Author Contribution:}

Author 1: This author wrote and edited the case report.

Author 2: This author was clinically involved with the case and edited the case report.

Author 3: This author was clinically involved with the case and edited the case report.

Author 4: This author was clinically involved with the case and edited the case report.

Author 5: This author was clinically involved with the case and edited the case report.

\section{References:}

1. Shen KR. Management of acquired nonmalignant tracheoesophageal fistula: Surgical pearls. The Journal of Thoracic and Cardiovascular Surgery . 2017;154(6):e123. doi: 10.1016/j.jtcvs.2017.08.031.

2. Yardeni D. Yardeni H. Coran AG. et al. Severe esophageal damage due to button battery ingestion: Can it be prevented? Pediatr Surg Int. 2004;(20):496-501.

3. Soto PH. Reid NE. Litovitz TL. Time to perforation for button batteries lodged in the esophagus. Am $J$ Emerg Med . 2019;(37):805-9.

4. Ahmad NS. Dobby N. Walker E. et al. A multicenter audit of the use of bronchoscopy during open and thoracoscopic repair of esophageal atresia with tracheoesophageal fistula. Pediatric Anesthesia . 2019;29(6):640-647. doi: 10.1111/pan.136219.

5. Buttazzoni E. Gregori D. Paoli B. et al. Susy Safe Working Group, Symptoms associated with button batteries injuries in children: An epidemiological review. Int $J$ Pediatr Otorhinolaryngol . $2015 ;(79): 2200-7$. 


\section{Figure legend:}

Figure 1. Tracheoesophageal Fistula. Tracheal View Towards Esophagus.

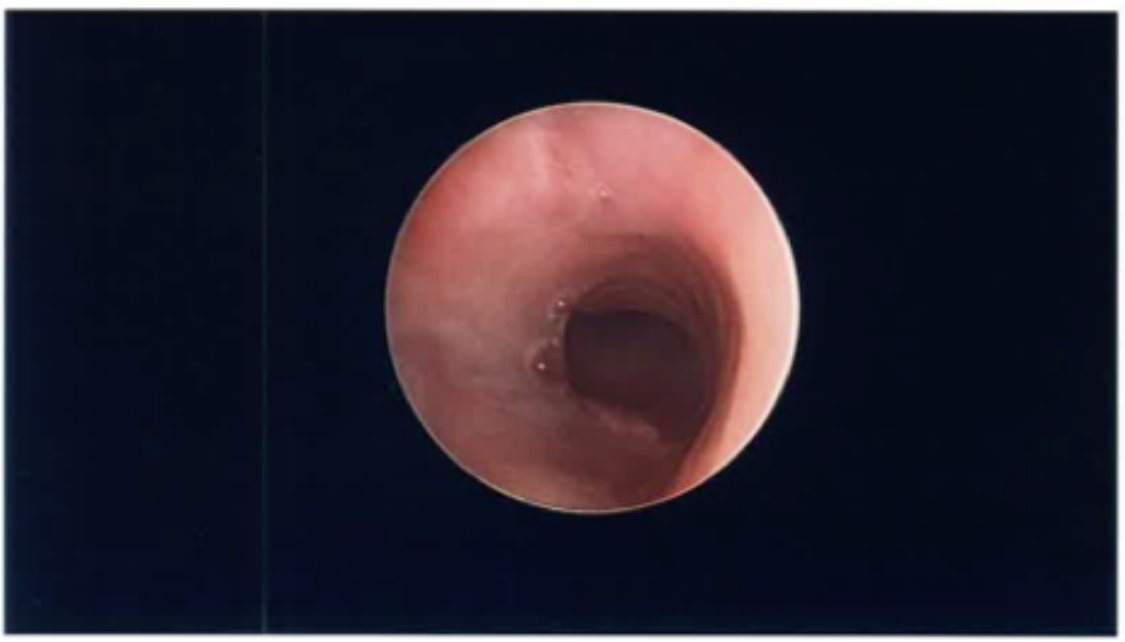

